INPLASY

PROTOCOL

To cite: Wang et al.

Comparison of efficacy of Traditional Chinese medicine capsules in the treatment of acute cerebral infarction: a net-like meta-analysis of randomized controlled trials. Inplasy protocol 2021110053. doi:

10.37766/inplasy2021.11.0053

Received: 15 November 2021

Published: 15 November 2021

Corresponding author:

Huihui Wang

2290994154@qq.com

Author Affiliation:

Nanfang Hospital, Southern

Medical University.

Support: Nanfang Hospital.

Review Stage at time of this submission: The review has not yet started.

Conflicts of interest:

None declared.

\section{Comparison of efficacy of Traditional Chinese medicine capsules in the treatment of acute cerebral infarction: a net-like meta-analysis of randomized controlled trials}

Wang, $\mathrm{H}^{1}$; Yang, Z2; Xu, S3.

Review question / Objective: To judge the efficacy of Traditional Chinese medicine capsules in the treatment of acute cerebral infarction by a net-like meta-analysis of randomized controlled trials. P:Patients with acute cerebral infarction (without other diseases complicated with acute cerebral infarction). I:Traditional Chinese medicine capsules (including Chinese medicine capsule combined with other treatment). C:Other treatment (including Other simple or combined non-capsule drug therapy). O:Chinese medicine capsule preparation is effective or ineffective in treating acute cerebral infarction. S:A net-like meta-analysis of randomized controlled trials.

Condition being studied: Acute cerebral infarction.Traditional Chinese medicine capsules. A net-like meta-analysis of randomized controlled trials.

INPLASY registration number: This protocol was registered with the International Platform of Registered Systematic Review and Meta-Analysis Protocols (INPLASY) on 15 November 2021 and was last updated on 15 November 2021 (registration number INPLASY2021110053).

\section{INTRODUCTION}

Review question / Objective: To judge the efficacy of Traditional Chinese medicine capsules in the treatment of acute cerebral infarction by a net-like meta-analysis of randomized controlled trials. P:Patients with acute cerebral infarction(without other diseases complicated with acute cerebral infarction). I:Traditional Chinese medicine capsules(including Chinese medicine capsule combined with other treatment). 
C:Other treatment(including Other simple or combined non-capsule drug therapy). $0:$ Chinese medicine capsule preparation is effective or ineffective in treating acute cerebral infarction. S:A net-like metaanalysis of randomized controlled trials.

Condition being studied: Acute cerebral infarction.Traditional Chinese medicine capsules.A net-like meta-analysis of randomized controlled trials.

\section{METHODS}

Participant or population: Icluding: Adults witih acute cerebral infarction. Exclusion:The date were incomplete, study with less than 20 patients, and other diseases complicated with acute cerebral infarction

Intervention: Traditional Chinese medicine capsules(including Chinese medicine capsule combined with other treatment).

Comparator: Traditional Chinese medicine capsules(including Chinese medicine capsule combined with other treatment)

Study designs to be included: We electronically searched the Cochrane Library (CENTRAL),PubMed, EMBASE, Chinese National Knowledge Infrastructure (CNKI), VIP Journals Database and Wanfang database until October 2021. Search terms included: (Traditional Chinese medicine) AND (Acute stroke OR Acute cerebral infarction OR Acute cerebral embolism). Chinese Databases were also searched using the above search terms in Chinese. We manually searched for a list of Chinese and English journals that may publish potentially eligible studies and checked the reference lists of all included studies and relevant review articles as well as.

Eligibility criteria: Icluding: Adults witih acute cerebral infarction.Exclusion:The date were incomplete, study with less than 20 patients, and other diseases complicated with acute cerebral infarction.
Information sources: We electronically searched the Cochrane Library (CENTRAL),PubMed, EMBASE, Chinese National Knowledge Infrastructure (CNKI), VIP Journals Database and Wanfang database until October 2021.

Main outcome(s): Comparison of curative effect of Chinese medicine capsule in treating acute cerebral infarction.

Additional outcome(s): None.

Quality assessment / Risk of bias analysis: The literature quality evaluntion mothod adopts the 12 criteria recommended by the Cochrane Back Review Group 16, which is evaluted independently by two participants. If the conclusions are inconsistent. the final decision will be made by the third-party experts.

Strategy of data synthesis: Data were analyzed using Review Manager (version 5.0).A fixed-effects model or randomeffects model was used to investigate the effects of RRR-based prescriptions on ischemic stroke across trials, and the weighted mean difference (WMD) was calculated for continuous outcomes, where as relative risk (RR) and $95 \%$ confidence intervals were calculated for dichotomous outcomes. Heterogeneity between trial results was tested using a standard chisquare test and 12 statistic was also calculated. Publication bias was graphically represented using a funnel plot. Two-tailed $p$ val-ues less than 0.05 were considered statistically significant.

Subgroup analysis: Subgroup analysis included the name of Chinese medicine preparation, treatment method and time of recovery, etc.

Sensitivity analysis: The main methods of sensitivity analysis include changing the inclusion criteria (especially for controversial studies), excluding lowquality studies, and using different statistical methods/models to analyze the same data.

Language: English. 
Country(ies) involved: China.

Keywords: acute cerebral infarction. Traditional Chinese medicine capsules. A net-like meta-analysis of randomized controlled trials.

Contributions of each author:

Author 1 - Huihui wang.

Author 2 - Zhen Yang.

Author 3 - Shan Xu. 